\title{
ESTUDIO DE DISTRIBUCIÓN DE TIEMPOS DE RESIDENCIA EN UN REACTOR BIOLÓGICO DE LECHO EMPACADO CERÁMICO
}

Tatiana Rodríguez Chaparro MSc trodri@umng.edu.co

Ingeniera civil. Magíster en ingeniería civil-ambiental Universidad de los Andes

Docente-Investigadora

Universidad Militar Nueva Granada. Programa de Ingeniería Civil. Bogotá-Colombia.

Luz Helena Pinzón. Ingeniera civil, egresada de la Universidad Militar Nueva Granada.

Carlos Alberto Arámbula. Ingeniero civil, egresado de la Universidad Militar Nueva Granada.

Fecha de recepción: 21 de junio de 2004

Fecha de aprobación:21 de julio de 2004

\section{RESUMEN}

La distribución de tiempos de residencia de un reactor es una característica del mezclado que ocurre dentro de él [1] [2]; su determinación es básica para el diseño de cualquier tipo de reactor en escala real. El objetivo de esta investigación consistió en determinar la distribución de tiempos de residencia en un reactor biológico de lecho empacado cerámico (anillos) a partir de pruebas con trazadores. Los resultados obtenidos utilizando las técnicas de inyección por paso y pulso fueron 34.577 seg., y 17.745 seg., respectivamente, y la dispersión calculada infinita. Lo anterior permite concluir que en reactores de lecho empacado cerámico (anillos) las moléculas del trazador se distribuyen uniformemente en todo el sistema. Los ensayos se realizaron en un modelo a escala laboratorio.

Palabras clave: dispersión, lecho empacado cerámico, paso, pulso, reactor, varianza

\section{ABSTRACT}

Residence time distribution in a reactor is a characteristic of the mixing process taking place within [1] [2]; its determination is basic for the actual design of any type of reactor The objective of this research is the determination of the residence time distribution in a biological packed bed reactor using standard tracer tests. The results using the techniques of injection by step and pulse indicated and average residence time of $34.577 \mathrm{sec}$., and $17.745 \mathrm{sec}$. respectively, and the calculated dispersion is infinite. The previous observation allows us to conclude that in a particular biological packed bed reactor the tracer molecules are distributed uniformly in the entire system. The tests were conducted on a laboratory scale unit.

Key words: dispersion, ceramic packing bed, step, pulse, reactor, variance

\section{INTRODUCCIÓN}

La teoría y experimentación que existen en ingeniería química sobre diseño de reactores necesariamente deben ser el punto de partida para el diseño de reactores biológicos 
(tanques de aireación) en ingeniería sanitaria; determinar cuánto tiempo duran las moléculas del fluido en el sistema y cómo es el comportamiento del flujo es fundamental para diseñar, construir y operar el reactor apropiado para cada tipo de agua residual.

\section{MATERIALES Y MÉTODOS}

El reactor estudiado como se observa en las figuras 1,2,3 y 4 está compuesto por 2 unidades con lecho empacado de anillos cerámicos de diferentes diámetros (ver tabla 1) dispuestos organizadamente en filas y columnas.

Tabla 1. Especificaciones técnicas del reactor biológico de lecho empacado cerámico

\begin{tabular}{|c|c|c|c|c|c|}
\hline Unidades & $\begin{array}{c}\text { Diámetro } \\
\text { anillos } \\
\text { cerámicos } \\
(\mathrm{cm})\end{array}$ & $\begin{array}{c}\text { Longitud } \\
\text { anillos } \\
\text { cerámicos } \\
(\mathrm{cm})\end{array}$ & $\begin{array}{c}\text { Profundidad } \\
\text { del lecho } \\
\text { empacado } \\
(\mathrm{cm})\end{array}$ & $\begin{array}{c}\text { Diámetro } \\
\text { del } \\
\text { reactor } \\
(\mathrm{cm})\end{array}$ & $\begin{array}{c}\text { Volumen } \\
\text { de agua } \\
\text { por tratar } \\
(\mathrm{I})\end{array}$ \\
\hline 1 & 2.5 & 4.5 & 30 & 15.25 & 12 \\
\hline 2 & 1.5 & 3.0 & 30 & 15.25 & 12 \\
\hline
\end{tabular}

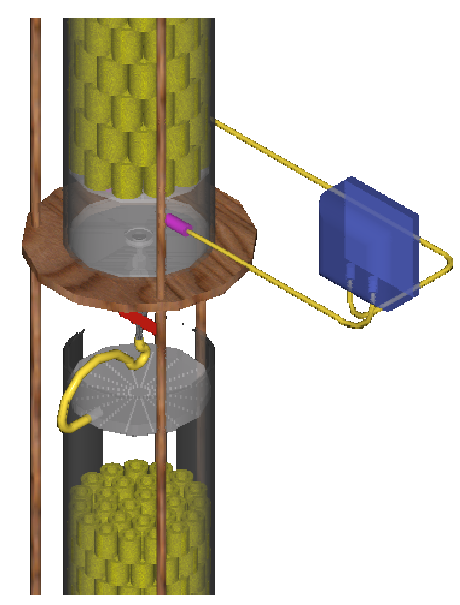

Figura 1. Reactor de lecho empacado cerámico (anillos)

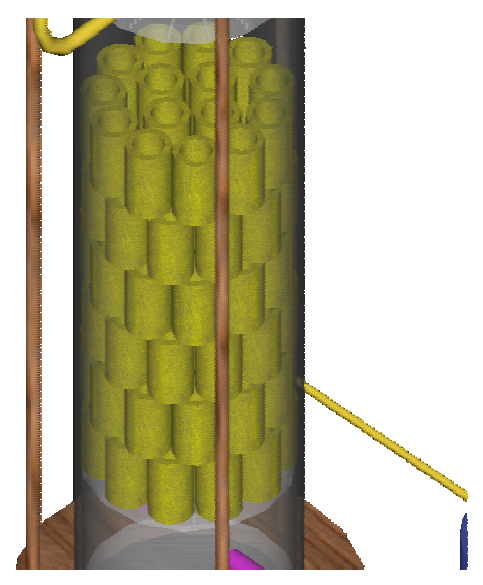


Figura 2. Reactor 1. (anillos grandes)

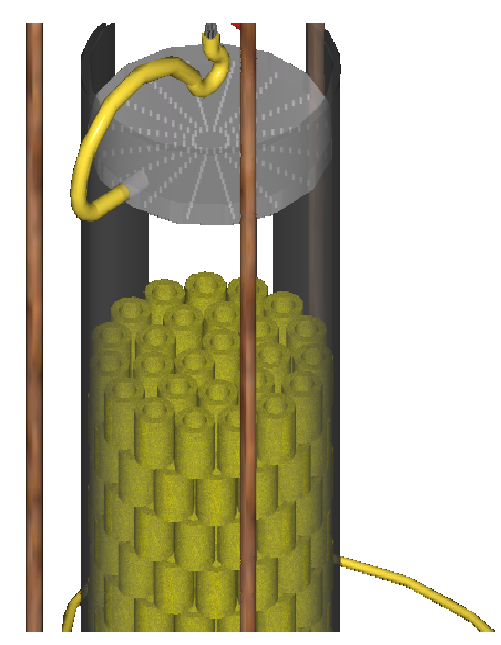

Figura 3. Reactor 2. (anillos pequeños)

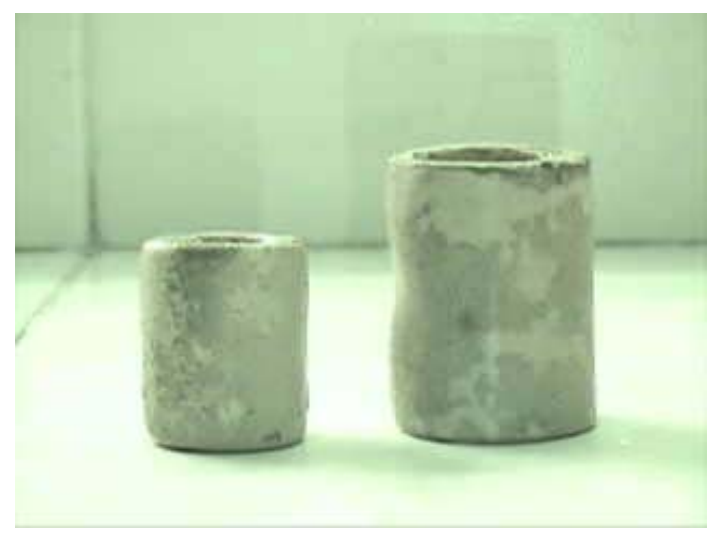

Figura 4. (anillos cerámicos)

La determinación del tiempo de residencia medio $\left(t_{m}\right)$ se realizó experimentalmente por medio de una prueba con trazadores, utilizando cloruro de potasio como trazador con concentración inicial conocida, inyectado por pulso y por paso en el instante $t=0$. 


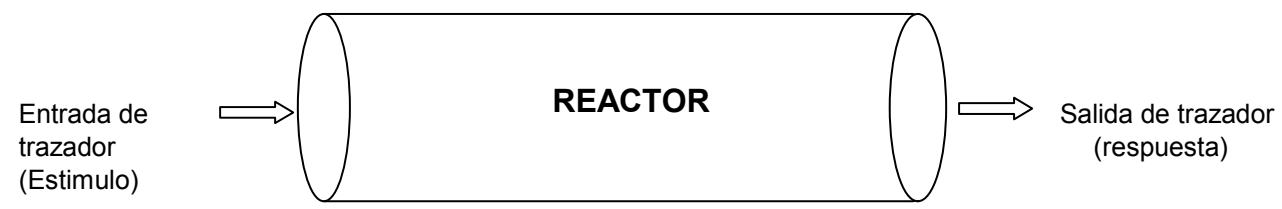

(Estimulo)

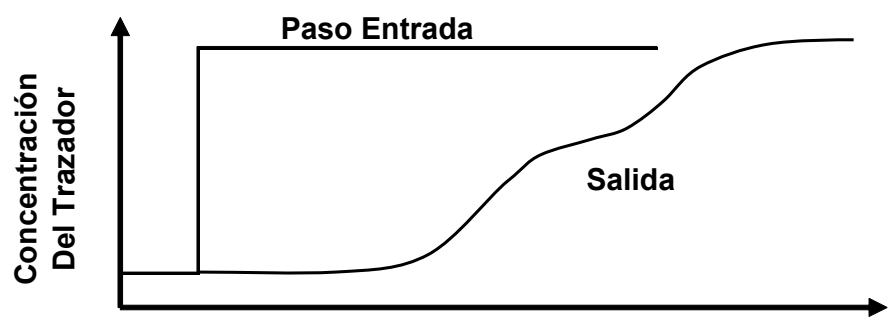

Tiempo

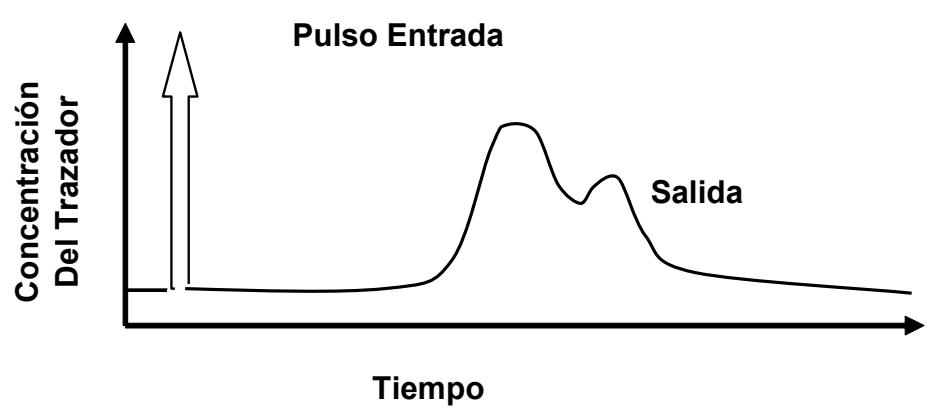

Figura 5. Técnicas de estímulo-respuesta normalmente utilizadas para estudiar el flujo en reactores. Fuente: Fogler [1]

Se realizaron 10 ensayos con diferentes intervalos de tiempo $(\Delta t=15 \mathrm{seg}, \Delta t=20 \mathrm{seg})$ en cada una de las unidades y en el reactor completo (ver tabla 2), la metodología para determinar el tiempo de residencia medio es la expuesta por Fogler [1] ( ver tabla 3).

Tabla 2. Secuencia de toma de datos en la prueba con trazadores

\begin{tabular}{|c|c|c|c|}
\hline Unidades & Técnica & Concentración & $\Delta t$ (seg) \\
\hline
\end{tabular}




\begin{tabular}{|c|c|c|c|}
\hline & utilizada & de trazador & \\
\hline Reactor 1 & \multirow{2}{*}{ pulso } & $30 \mathrm{mg} / \mathrm{l}$ & 20 \\
\cline { 1 - 1 } Reactor 1 & & & 15 \\
\hline Reactor 1 & paso & & 15 \\
\hline Reactor 1 & pulso & & 20 \\
\hline Reactor 2 & & \multirow{4}{*}{$50 \mathrm{mg} / \mathrm{l}$} & 15 \\
\hline Reactor 2 & paso & & 15 \\
\hline Reactor 2 & pulso & & 20 \\
\hline Reactor completo & \multirow{2}{*}{ pulso } & & 15 \\
\hline Reactor completo & & & 20 \\
\hline Reactor completo & paso & & 15 \\
\hline
\end{tabular}

Tabla 3. Metodología para determinar la distribución de tiempos de residencia y la dispersión en un reactor.

\begin{tabular}{|c|c|c|}
\hline Paso & Ecuación & Observaciones \\
\hline 1 & $C(t)$ & $\begin{array}{l}\text { Inyección del trazador en un instante } t=0 \text { y } \\
\text { medición de la concentración }(c) \text { en el efluente en } \\
\text { función del tiempo }\end{array}$ \\
\hline 2 & $E(t)=\frac{C(t)}{\int_{0}^{\infty} C(t) d t}$ & $\begin{array}{l}\text { Construcción de las curvas } c(t) \text { y } E(t) \text {. La curva } \\
E(t) \text { indica en forma numérica el tiempo que } \\
\text { tardan las partículas del fluido en el reactor. }\end{array}$ \\
\hline 3 & $t_{m}=\frac{\int_{0}^{\infty} t \cdot E(t) d t}{\int_{0}^{\infty} E(t) d t}, t_{m}=\int_{0}^{\infty} t \cdot E(t) d t$ & $\begin{array}{l}\text { Cálculo del área bajo la curva de la grafica } \\
t . E(t) v s t \text {; este resultado corresponde al tiempo de } \\
\text { residencia medio }\left(t_{m}\right)\end{array}$ \\
\hline 4 & $\sigma^{2}=\int_{0}\left(t-t_{m}\right)^{2} \cdot E(t) d t$ & $\begin{array}{l}\text { Cálculo del área bajo la curva de la gráfica; este } \\
\text { resultado corresponde a la varianza } \sigma^{2} \text {. }\end{array}$ \\
\hline 5 & $\frac{\sigma^{2}}{t_{m}^{2}}=\frac{2}{p e}-\frac{2}{p e^{2}} *\left(1-p e^{-p e}\right)$ & $\begin{array}{l}\text { Cálculo de la dispersión utilizando el método de la } \\
\text { varianza, que relaciona } \sigma^{2} \text { con pe donde } P e= \\
\text { número de Peclet }=\frac{u L}{D} \text { donde: } u=\text { velocidad } \\
\text { tomada a partir de un caudal, } L=\text { Longitud del } \\
\text { reactor y } D=\text { coeficiente dispersión. }\end{array}$ \\
\hline
\end{tabular}

\section{RESULTADOS Y DISCUSIÓN}

Los resultados obtenidos a partir de la metodología expuesta anteriormente se pueden observar en la Tabla 4. 
Tabla4. Resultados de la prueba con trazadores

\begin{tabular}{|c|c|c|c|c|c|c|c|c|}
\hline \multirow[t]{2}{*}{ Unidades } & \multirow{2}{*}{$\begin{array}{c}\text { Concentración } \\
\text { de } \\
\text { trazador }\end{array}$} & \multirow[t]{2}{*}{$\Delta t$ (seg.) } & \multicolumn{2}{|c|}{$t_{m}$ (seg.) } & \multicolumn{2}{|c|}{$\begin{array}{l}\text { Relación } \\
\sigma^{2} / t_{m}{ }^{2}\end{array}$} & \multicolumn{2}{|c|}{$\begin{array}{c}\text { Número } \\
\text { de Peclet } \\
P e=u L / D\end{array}$} \\
\hline & & & Pulso & Paso & Pulso & Paso & Pulso & Paso \\
\hline Reactor 1 & $30 \mathrm{mg} / \mathrm{l}$ & 20 & 7.736 & & 31.024 & & \multirow{10}{*}{$\infty$} & \multirow{10}{*}{$\infty$} \\
\hline Reactor 1 & $50 \mathrm{mg} / \mathrm{l}$ & 15 & 10.631 & & 22.548 & 16.002 & & \\
\hline Reactor 1 & $50 \mathrm{mg} / \mathrm{l}$ & 15 & & 20.372 & & & & \\
\hline Reactor 1 & $50 \mathrm{mg} / \mathrm{l}$ & 20 & 8.210 & & 27.861 & & & \\
\hline Reactor 2 & $50 \mathrm{mg} / \mathrm{l}$ & 15 & 9.734 & & 19.886 & & & \\
\hline Reactor 2 & $50 \mathrm{mg} / \mathrm{l}$ & 15 & & 21.483 & & 15.694 & & \\
\hline Raector 2 & $50 \mathrm{mg} / \mathrm{l}$ & 20 & 8.373 & & 29.316 & & & \\
\hline $\begin{array}{c}\text { Reactor } \\
\text { completo }\end{array}$ & $50 \mathrm{mg} / \mathrm{l}$ & 15 & 18.893 & & 26.013 & & & \\
\hline $\begin{array}{c}\text { Reactor } \\
\text { completo }\end{array}$ & $50 \mathrm{mg} / \mathrm{l}$ & 20 & 16.597 & & 19.263 & & & \\
\hline $\begin{array}{l}\text { Reactor } \\
\text { completo }\end{array}$ & $50 \mathrm{mg} / \mathrm{l}$ & 15 & & 34.577 & & 15.176 & & \\
\hline
\end{tabular}

Como se observa, el tiempo de residencia medio $(\mathrm{tm})$ de las partículas en el trazador en el reactor 1 por pulso es de 8.859 seg., por paso de 20.372 seg., en el reactor 2 por pulso 9.054 seg., por paso $21.483 \mathrm{seg}$., y en el reactor completo por pulso $17.745 \mathrm{seg}$ y por paso 34.577seg.; así mismo utilizando el método para calcular la dispersión que relaciona el número de Peclet con el tiempo de residencia promedio y la varianza, se encontraron valores tendientes a infinito en todos los ensayos.

Los resultados en la presente investigación indican que la ecuación $\frac{\sigma^{2}}{t_{m}{ }^{2}}=\frac{2}{P e}-\frac{2}{P e^{2}} *\left(1-e^{-P e}\right)$ sólo se puede emplear cuando los valores de la relación varianza/tiempo promedio de residencia están en el rango de 0-1.

Las figuras 5-14 ilustran las curvas empleadas para calcular los tiempos de residencia promedio; así mismo se muestra el ajuste realizado por medio de un modelo polinómico de sexto grado que para todos los ensayos presentó valores de correlación $\left(r^{2}\right)$ superiores a 0.96 . 


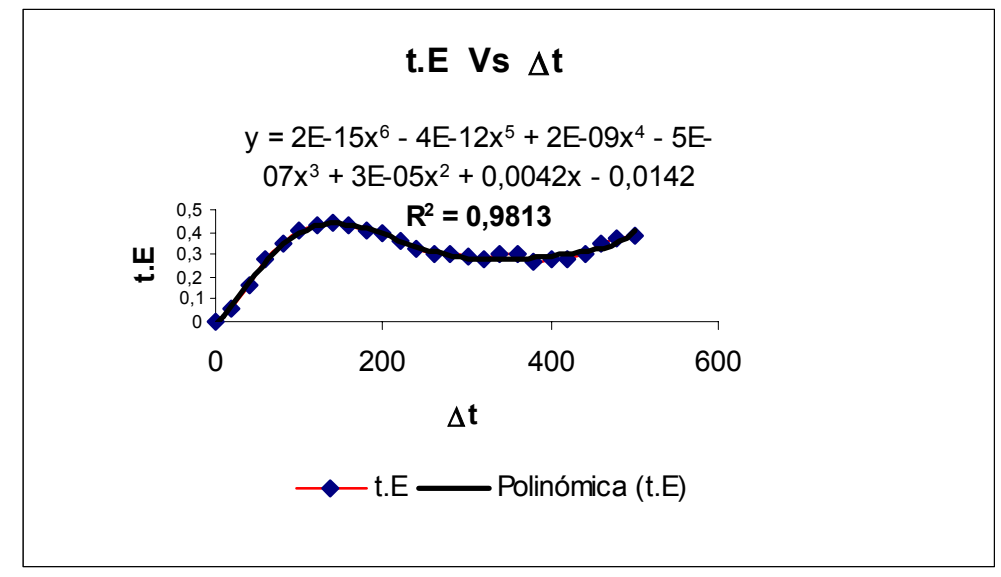

Figura 5. t.E vs t en el reactor 1 con $\Delta \mathrm{t}$ de 20 seg. por inyección de pulso

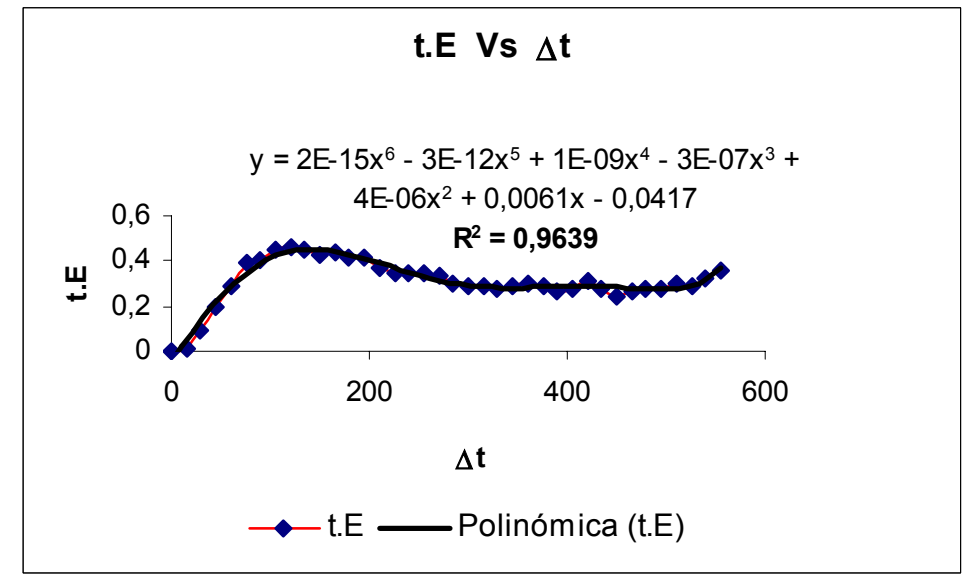

Figura 6. t.E vs t en el reactor 1 con $\Delta \mathrm{t}$ de 15 seg. por inyección de pulso

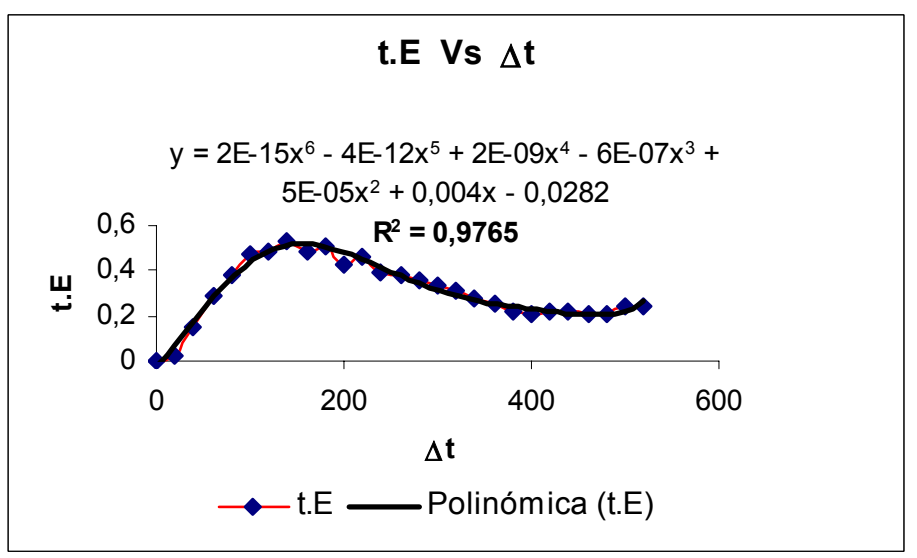

Figura 7. t.E vs t en el reactor 1 con $\Delta \mathrm{t}$ de 20 seg. por inyección de pulso 


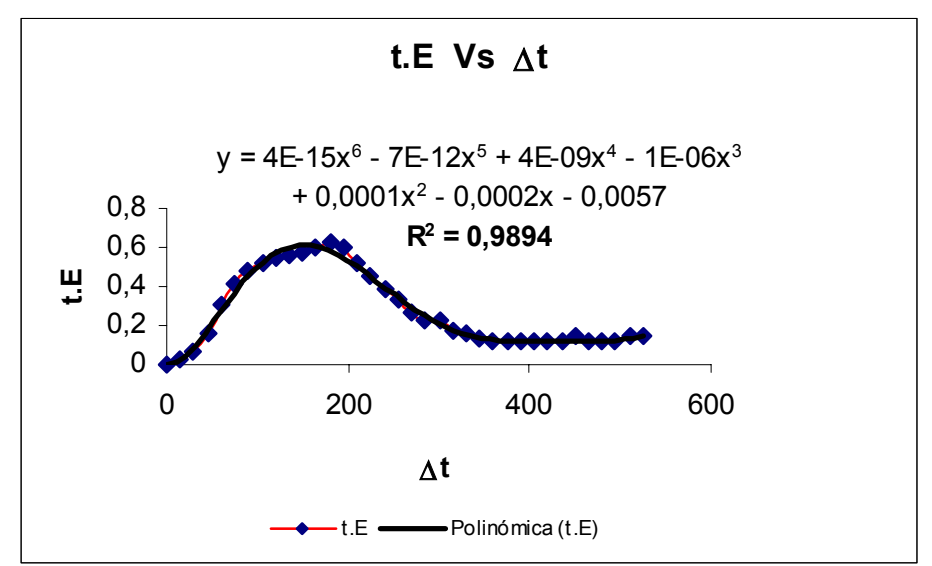

Figura 8. t.E vs t en el reactor 2 con $\Delta \mathrm{t}$ de 15 seg. por inyección de pulso

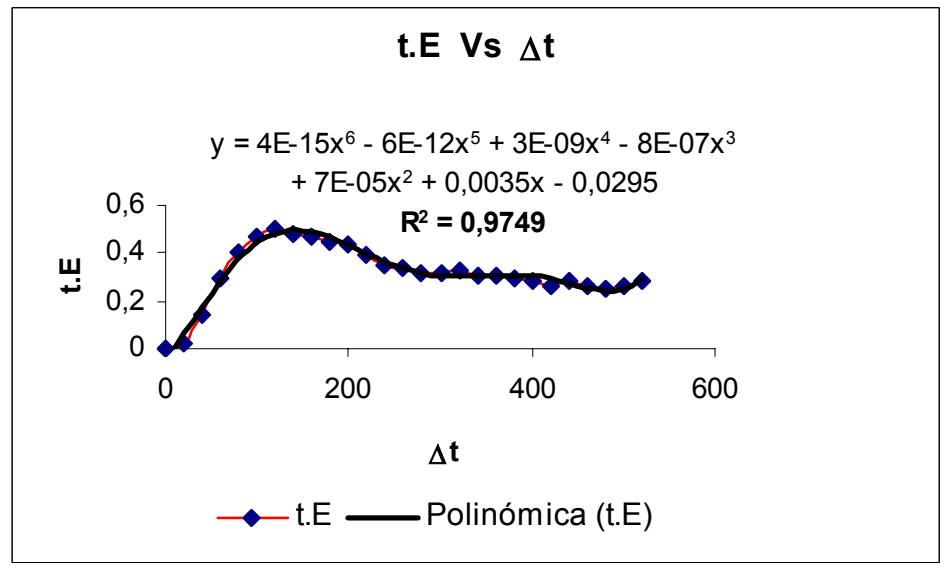

Figura 9. t.E vs t en el reactor 2 con $\Delta \mathrm{t}$ de 20 seg. por inyección de pulso

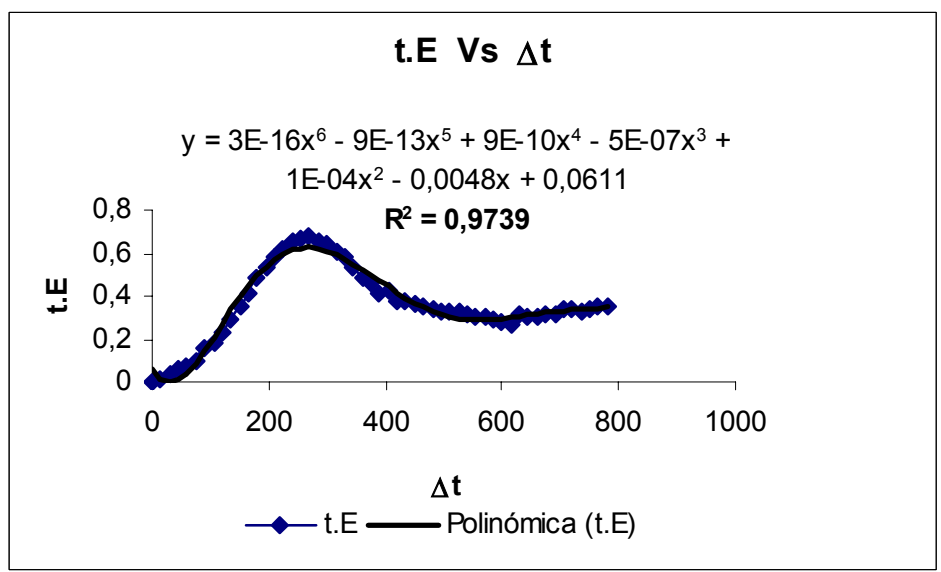

Figura 10. t.E vs $t$ en el reactor completo con $\Delta t$ de 15 seg. por inyección de pulso 


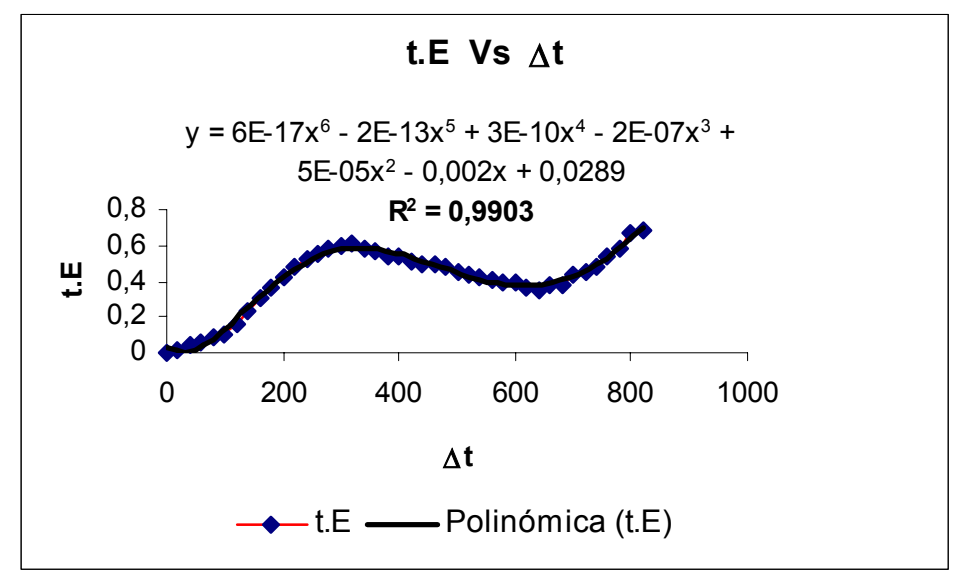

Figura 11. t.E vs t en el reactor completo con $\Delta \mathrm{t}$ de 20 seg. por inyección de pulso

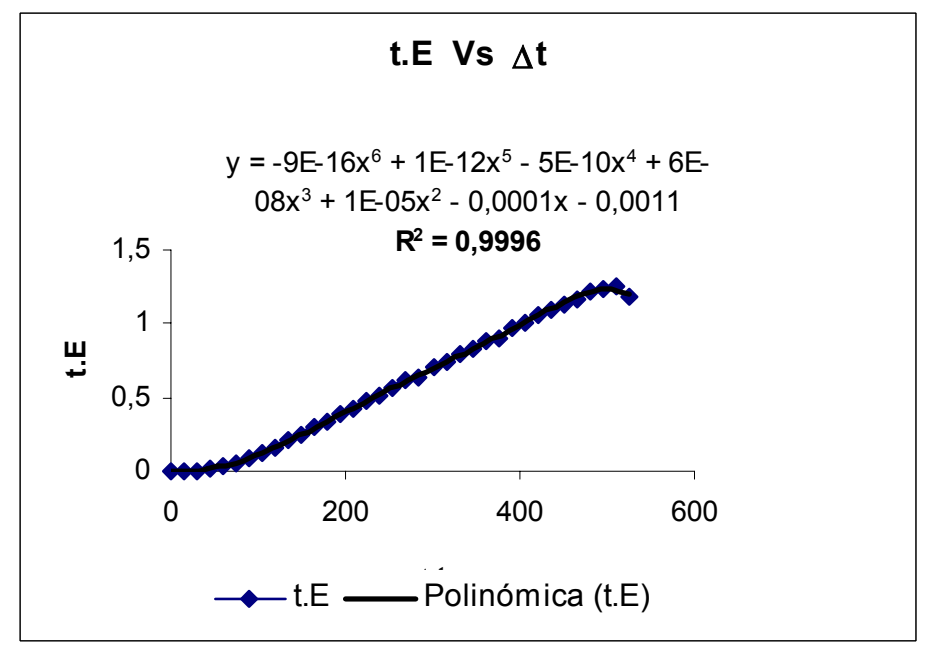

Figura 12. t.E vs t en el reactor 1 con $\Delta$ t de 15 seg. por inyección de paso

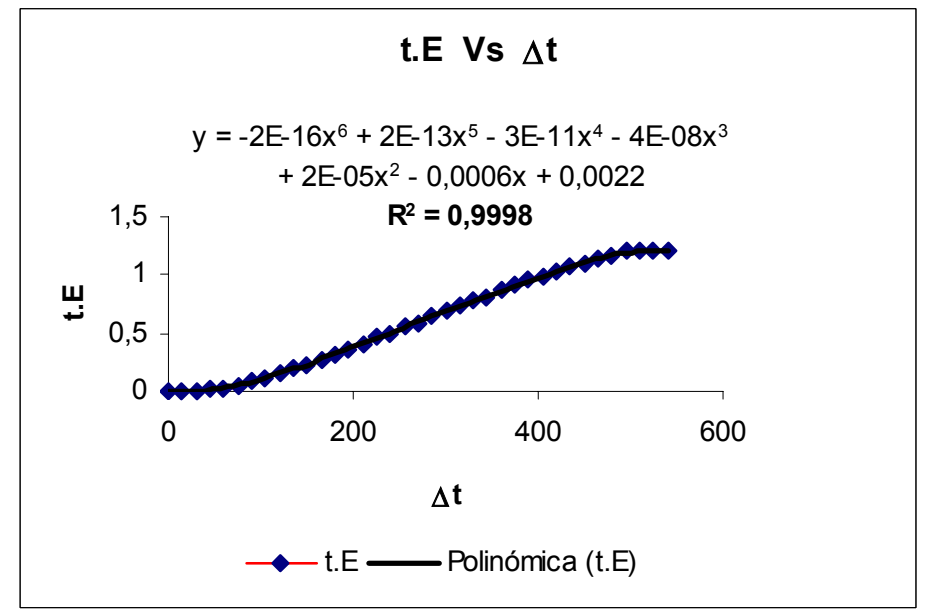

Figura 13. t.E vs t en el reactor 2 con $\Delta t$ de 15 seg. por inyección de paso 


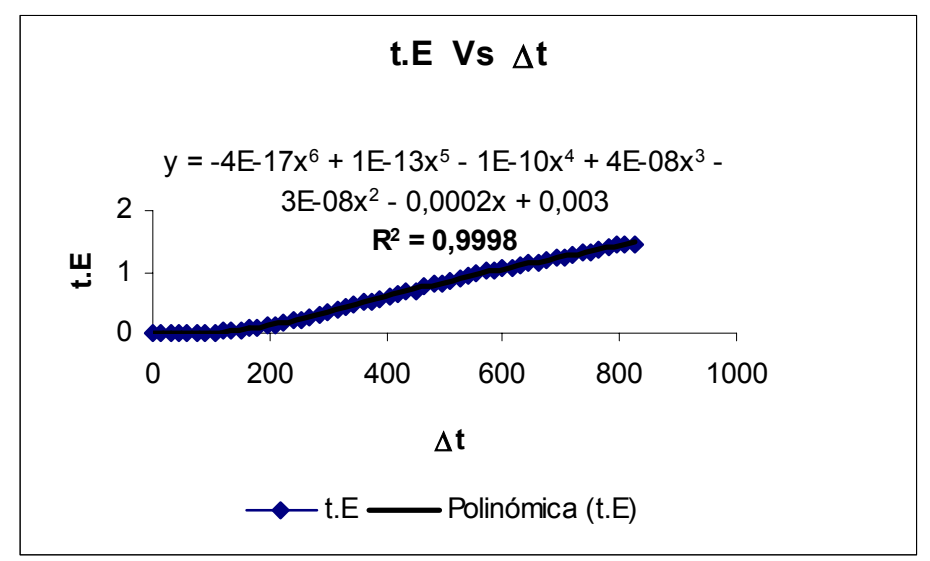

Figura 14. t.E vs t en el reactor completo con $\Delta \mathrm{t}$ de 15 seg. por inyección de paso

\section{CONCLUSIONES}

El tiempo de residencia medio $\left(t_{m}\right)$ calculado a partir de la prueba con trazadores, realizada con cloruro de potasio fue de 17.745 seg., y 34.577 seg. por inyección de pulso y paso respectivamente.

El método para determinar la dispersión de las partículas en el sistema relacionando, el tiempo de residencia promedio, la varianza y el número de Peclet no es apropiado para reactores de dimensiones pequeñas y cuyo valor $\frac{\sigma^{2}}{t_{m}{ }^{2}}$ sea $>1$.

Las graficas t.Evs $\Delta t$ de las cuales se obtiene el tiempo de residencia promedio se ajustaron a un modelo polinomico de sexto grado dando correlaciones $\left(r^{2}\right)$ superiores a 0.96 en todos los casos.

Debido a que la relación $\frac{u L}{D}$ tiende a $\infty$ se puede concluir que la dispersión en el reactor se asemeja al comportamiento que tienen las partículas en reactores de flujo completamente mezclado.

\section{AGRADECIMIENTOS}

Los autores agradecen la colaboración para el desarrollo de esta investigación al Dr. Watson Vargas, al laboratorista Jesús Ramos y al Centro de Investigaciones de la Facultad de Ingeniería de la Universidad Militar Nueva Granada. 


\section{REFERENCIAS BIBLIOGRÁFICAS}

[1] Fogler, h., (2001). Elementos de Ingeniería de las Reacciones Químicas. México, Pearson, 809-870 $p$

[2] Levenspiel, o., (1962). Chemical Reaction Engineering. New York, Ed. Jhon Wiley and Sons, 253-309 $p$ 